\title{
Herd-level prevalence of Johne's disease in Utah and adjacent areas of the Intermountain West as detected by a bulk-tank milk surveillance project
}

\author{
D. J. Wilson, ${ }^{* 1}$ K. Rood, ${ }^{*}$ P. Biswas, $†$ and T. M. Byrem $\dagger$ \\ *Department of Animal, Dairy and Veterinary Sciences, Utah State University, Logan 84321 \\ †Antel BioSystems, Lansing, MI 48910
}

\begin{abstract}
The objectives of this study were to estimate the dairy herd-level prevalence of Johne's disease (JD) in Utah and nearby areas of the Intermountain West and to estimate the sensitivity of a single bulk-tank milk test for JD detection. Two milk samples from all bulk tanks on the study farms were collected 1 mo apart. Samples were frozen and shipped to a laboratory for JD testing. An ELISA to measure total IgG antibody specific against Mycobacterium avium ssp. paratuberculosis, the etiological agent that causes JD, and a quantitative real-time PCR to detect $M$. avium ssp. paratuberculosis DNA were used; both tests were designed for bulk milk. Of the dairy farms in the study area, 170/246 (69\%) participated. Positive JD results were found in bulk milk from $67 / 170$ (39\%) of dairy farms in Utah and adjacent areas. There were 138 JD-positive bulk-tank results from 241 bulk-tank samples from the 67 positive herds. The sensitivity of the bulk milk testing for detection of JD was 138/241(57\%). From the 103 JD-negative farms, 235 bulk-tank samples tested negative for JD. The probability of false-negative results on a single bulk-milk sample was $(1-0.57)=0.43$. For farms with 1 bulk tank, 2 samples collected 1 mo apart, with both samples testing negative (by both ELISA and quantitative real-time PCR) for JD, the true-negative probability was $\left[1-(0.43)^{2}\right]=(1-0.18)=82 \%$. For farms with at least 2 bulk tanks, at least 4 samples tested, with all results negative for JD, the true-negative probability was at least $97 \%$. Results support other estimates that prevalence of JD has increased over the last 15 to 20 yr. However, the prevalence detected was 3 times that from a recent report where $13 \%$ of dairy herds in the western US were positive. The increase in JD suggests that current control programs, at least as applied, are not effective. Bulk milk testing is a practical way to screen dairy herds for presence of JD. Studies are
\end{abstract}

Received May 27, 2010.

Accepted September 10, 2010.

${ }^{1}$ Corresponding author: david.wilson@usu.edu needed regarding the use of individual cow milk tests for accuracy, practicality, and effectiveness in reducing the prevalence of JD in dairy herds.

Key words: dairy cattle, epidemiology, Johne's disease, milk

\section{INTRODUCTION}

Johne's disease (JD; paratuberculosis) is a chronic and important digestive-tract disease in dairy cattle. Affected animals may exhibit no clinical signs, or may exhibit milk production loss, weight loss progressing to emaciation, intermittent or chronic diarrhea, or death. Cows with JD are also at increased risk of culling (Wilson et al., 1993; Stabel, 1998; Tiwari et al., 2005, 2008; Clark et al., 2008). The etiologic agent is Mycobacterium avium ssp. paratuberculosis (MAP; Stabel, 1998).

Johne's disease is costly to the dairy industry. Financial loss caused by JD has been estimated at $\$ 83$ to $\$ 248$ in lost milk per infected cow depending on parity or age, beginning in second lactation, along with $\$ 77$ in increased culling losses per cow for all cows within a herd with a JD prevalence of $21 \%$ (Wilson et al., 1995); $\$ 100$ to $\$ 200$ per cow for all cows within infected herds (Ott et al., 1999); \$49 per cow for all cows within infected herds if there were 50 lactating cows (Chi et al., 2002); or $\$ 380$ per case (Tiwari et al., 2008).

Testing for the disease is not performed in the vast majority of dairy herds in the United States, or it is done only intermittently on select cows, such as those suspected to be affected by JD based on clinical signs (USDA APHIS, 2008). In Utah, approximately 1\%, or 3 of the 265 dairy farms in the state, did any testing for JD in 2007 (W. Hess, Utah Department of Agriculture and Food, Salt Lake City, UT, personal communication). Based on the limited information available, estimates of the percentage of dairy herds with JD continue to increase in the United States and Canada, from approximately 10 to $30 \%$ of herds 15 to $20 \mathrm{yr}$ ago (Merkal et al., 1987; Collins et al., 1994; Sockett, 1996) to 40 to $74 \%$ of herds at present, with most regional estimates at approximately $43 \%$ of dairy herds with JD (Tiwari et al., 2006; Tavornpanich et al., 2008). However, according to the 2008 Animal and Plant Health 
Inspection Services report, $13 \%$ of dairy herds in the western United States were confirmed positive for JD in 2007 (USDA APHIS, 2008). The percentage of dairy cows in the United States or Canada infected with JD was estimated at 3 to $5 \% 15$ to $20 \mathrm{yr}$ ago (Merkal et al., 1987; Collins et al., 1994), and is estimated at 13 to $23 \%$ of dairy cows at present (McKenna et al., 2004; Pearce et al., 2008; Tiwari et al., 2008). It is speculated that JD prevalence is increasing, but current state or regional data from a high percentage of herds within a region is lacking.

The primary objective of this study was to estimate the dairy herd-level prevalence of JD in Utah and surrounding states in the Intermountain West. This is the first report of a surveillance project sampling the majority of dairy herds within a region of the United States to estimate the proportion of dairy herds infected with JD. Another objective was to estimate the sensitivity of a single bulk-tank milk test for detection of JD in a true-positive dairy herd.

\section{MATERIALS AND METHODS}

Written permission of dairy producers was required to sample bulk-tank milk for JD testing. Permission forms were distributed by e-mail and milk buyer field personnel visits to dairy producers in Utah and surrounding states that sold milk to either of the 2 major milk-buying companies in Utah.

\section{Collection and Handling of Bulk-Tank Milk Samples}

Two milk samples from all bulk tanks on all participating dairy farms were collected: 1 milk sample per bulk tank at each collection, collected 1 mo apart. Milk haulers collected an extra sample at the same time they collected their routine testing samples. All samples collected by the 2 milk buyers were frozen at $-20^{\circ} \mathrm{C}$ and were stored frozen without thawing at their milk-collection locations in freezers provided by the project. Using portable $-20^{\circ} \mathrm{C}$ freezers, all samples were transported to the Utah Veterinary Diagnostic Laboratory from the collection locations, and shipped frozen overnight by courier to Antel BioSystems (Lansing, MI) for testing.

\section{Testing for MAP in Bulk-Tank Milk Samples}

At Antel BioSystems, 2 separate analyses were performed on each bulk-tank sample as described previously (Biswas et al., 2008). Briefly, each bulk-tank sample was analyzed by ELISA for IgG antibody specific for MAP and for presence of MAP organism by quantitative real-time PCR (Q-PCR). For ELISA, 100 $\mu \mathrm{L}$ of whole milk sample was tested using a commer- cially available, preabsorbed, antibody-capture ELISA (Institut Pourquier, Montpellier, France). The assay was performed as described previously (van Weering et al., 2007) with the following exceptions: A 1:1.5 dilution of milk sample into sample diluent instead of $1: 2$, 1-h incubation times for sample and conjugate steps instead of $0.5 \mathrm{~h}, 1: 67$ dilution of concentrated conjugate instead of 1:100, and 20 min for color development instead of $10 \mathrm{~min}$ before stopping the reaction with 0.5 $M$ sulfuric acid. Positive and negative controls supplied with the ELISA kit yielded optical density values (420 $\mathrm{nm}$ ) between 2.3 and 3.0, and less than 0.10 , respectively. Scores for ELISA were calculated by subtracting negative control values from each sample value and comparing with a statistically optimal cut-off of 0.1 (Lombard et al., 2006; Biswas et al., 2008). The cut-off of 0.1 optical density, where ELISA scores $>0.1$ are considered positive for JD, yields a test sensitivity and specificity of 52 and $94 \%$, respectively, when compared with environmental fecal analysis as the "gold standard" for MAP infection in dairy herds (Lombard et al., 2006; Biswas et al., 2008).

For Q-PCR analysis for presence of MAP in milk, $50 \mathrm{~mL}$ of bulk milk was processed by centrifugation $(2,500 \times g)$, and total DNA was extracted from the pellet using a DNeasy spin kit (Qiagen Corp., Valencia, CA). The extracted DNA was subjected to Q-PCR using TaqMan chemistry (Applied Biosystems, Foster City, CA) and primers designed to detect the IS900 repeat element region in MAP genomic DNA. Cycle threshold values below 43 were considered positive for the presence of MAP genome, a threshold that yields sensitivity and specificity of 40 and $94 \%$, respectively, when compared with environmental fecal analysis for MAP infection in dairy herds (Lombard et al., 2006; Biswas et al., 2008).

Samples from the 2 milk buyers were coded by number for anonymity; the investigators did not know the dairy producers' identities. After testing was completed, owners of JD-positive herds were contacted through the milk buyer field personnel using their number code and their identities were revealed to the investigators. Two memoranda explaining the interpretation of testnegative and test-positive results were composed, and one was sent to all participating producers depending on whether all of their bulk-tank samples tested JDnegative or at least 1 sample tested positive for JD, respectively. Follow-up was offered to owners of all participating herds, including contact information for 2 of the authors (D. Wilson and K. Rood). The survey results also were presented at the major dairy producer meeting in Utah, attended by most producers in the state and some from surrounding states, in December 2009 . 


\section{Case Definitions and Statistical Methods}

The definition of "true positive" for JD in a herd was the finding of at least 1 positive test result for JD. Therefore, by definition, specificity was $100 \%$; no positive results were defined as "false positive." This is supported by previous reports that the specificity was almost 100\%; false-positive results are uncommon (Lombard et al., 2006; Biswas et al., 2008). Sensitivity was calculated by dividing the total number of JD-positive bulk-tank results (whether by either the ELISA or Q-PCR, or by both tests) by the total number of bulktank milk samples tested from the JD-positive herds. For example, if a farm had 2 bulk tanks, each sampled twice, 1 mo apart, resulting in 4 samples tested for JD and 2 of the 4 tank samples tested JD-positive, the sensitivity would be $2 / 4$, or $50 \%$. This calculation is likely to differ from calculating the sensitivity of a single test by either ELISA or Q-PCR. For example, if as stated above, a farm had 2 bulk tanks, each sampled twice 1 mo apart, resulting in 4 samples tested for JD, 4 ELISA and 4 Q-PCR tests were performed. Possibly 1 tank sample could be ELISA-positive, Q-PCR negative, another ELISA-negative and Q-PCR positive, and the remaining 2 samples negative by both tests. The sensitivity on each test basis would be $1 / 4$, or $25 \%$ (or if the sensitivity was calculated across all 8 tests, $2 / 8=$ $25 \%$ ), but the sensitivity on a bulk-tank sample results basis would be $2 / 4$, or $50 \%$.

Using sequential probability, the probability that any particular herd for which all tests were JD-negative was a "true negative" for JD was calculated. First, the probability of false-negative status was calculated as (1 sensitivity $)^{\mathrm{n}}$, where $\mathrm{n}=$ number of bulk tank milk tests performed. For example, if sensitivity was $57 \%$, and 1 bulk tank was sampled and tested twice for JD with both tests negative, the probability of false-negative status for the disease would be $(1-0.57)^{2}=(0.43)^{2}$ $=0.18$. Then, the probability of true negative for JD status can be calculated as $(1-0.18)=82 \%$.

Two alternative methods were used to calculate herdlevel prevalence of JD. The first calculation used all herds tested; prevalence was defined as the number of positive herds divided by the total number of herds tested. In the alternative analysis, herds with only 1 bulk-tank milk sample tested with negative results for JD were considered of unknown status and therefore excluded from calculation of prevalence by subtracting them from the denominator.

\section{RESULTS}

\section{Sample Collection}

Permission forms distributed by the 2 milk-buying companies reached 172 of the 246 dairy farms in Utah and the study areas adjacent to Utah in the Intermountain West (70\%). Signed forms authorizing participation in the project were returned by 170 producers, totaling $170 / 246(69 \%)$ of the dairy producers in the study area.

Bulk-tank milk samples $(\mathrm{n}=476)$ were collected from all bulk tanks on the 170 participating dairy farms, sampled twice 1 mo apart. All samples were frozen at $-20^{\circ} \mathrm{C}$ as described earlier. All samples were transported in the portable $-20^{\circ} \mathrm{C}$ freezers and shipped frozen overnight to the laboratory in Lansing, MI, where they arrived frozen.

\section{Herd-Level Prevalence}

Johne's disease was detected in bulk-tank milk by at least 1 test from 67/170 dairy farms (39\%) in Utah and adjacent study areas (Table 1). The remaining 103/170 (61\%) dairy farms were negative for JD on all bulk-tank milk samples tested (Table 1). The number of bulk-tank milk samples tested per farm ranged from 1 to 24 . Every farm should have had an even number of samples, with a minimum of 2 samples, but 27 farms inadvertently had only 1 sample collected from 1 bulk tank. Of those 27 single-sampled farms, 2 tested positive for JD. When the 25 herds with only 1 bulk-tank milk sample that tested negative for JD were excluded, the prevalence estimate was $67 / 145(46 \%)$. The most common test results among the 67 dairy farms defined as JD-positive were 1 or 2 positives $/ 4$ tests $(n=28$ farms, each with 1 bulk tank). The most extreme results in terms of proportion of tests that were JD-positive on particular farms were as follows: lowest proportions positive, 2/48 (a farm with 12 bulk tanks), 1/10 (3 farms), 1/8 (3 farms), 2/14, 2/12 (2 farms); highest proportions positive, $13 / 24,7 / 12,6 / 8,9 / 12,5 / 6,10 / 12,4 / 4,8 / 8$.

\section{Bulk-Tank Test Results}

Bulk-tank test results for the 476 tank samples are shown in Table 1. A total of 241 bulk-tank samples was collected from the 67 herds that tested positive for JD at least once. Of these, 138 samples tested positive for JD by 1 or both methods. This included 25 bulktank samples that were positive by both the ELISA and Q-PCR, 67 positive only by the ELISA, and 46 positive only by the Q-PCR. In addition, the remainder of the 241 tank samples from the 67 positive herds included 103 that tested negative by both the ELISA and Q-PCR. In addition, 235 bulk-tank samples, all of which tested negative for JD, were collected from the remaining 103 farms that were therefore defined as JD-negative. In total, 138 tank tests were JD-positive and 338 were JD-negative. 
Table 1. Herd-level and individual bulk-tank test results for presence of Johne's disease (JD) in 170 dairy herds in Utah and parts of surrounding states

\begin{tabular}{lccc}
\hline & & & \\
tem & JD & JD & \\
positive & negative & Total \\
\hline Herds, n (\%) & $67(39)^{1}$ & $103(61)^{2}$ & 170 \\
MAP ELISA positive only, n (\%) & $67(14)^{3}$ & 0 & \\
MAP quantitative real-time PCR positive only, n (\%) & $46(10)^{3}$ & 0 & \\
Positive by both tests, n (\%) & $25(5)^{3}$ & 0 & \\
Negative by both tests, n (\%) & $103(22)^{3}$ & $235(49)^{3}$ & \\
Total bulk tank milk samples, tanks & 241 & 235 & 476 \\
\hline
\end{tabular}

${ }^{1}$ Herds that tested positive for JD (Mycobacterium avium spp. paratuberculosis, MAP) at least once by 1 bulktank milk test.

${ }^{2}$ Herds that tested negative for JD (MAP) by all bulk-tank milk tests.

${ }^{3}$ Number (\%) of bulk tank milk samples that tested with this result out of all 476 bulk-tank samples.

\section{Agreement Between the Test Methods on the Same Bulk-Tank Sample}

Of the 952 JD tests performed on the 476 bulk tank samples in the project, both the ELISA and Q-PCR results on the same sample agreed 726 times: all 470 negative tests on all 235 tank samples from the 103 JD-negative herds, 206 negative tests from 103 tank samples from the 67 JD-positive herds, and 50 doublepositive tests from 25 tank samples from the 67 JDpositive herds (Table 2). The remaining 226 tests were performed on 113 tank samples with discordant results: 67 samples were positive only by the ELISA, and 46 were positive only by the Q-PCR. Therefore, the overall agreement between the bulk-tank ELISA and Q-PCR tests was 726/952 (76\%). Within JD-positive herds, the agreement between the 2 tests was 256/482 (53\%; Table 2).

\section{Bulk-Tank Test Sensitivity}

If test sensitivity were defined as the total number of positive test results divided by the total number of tests (both ELISA and Q-PCR) performed on bulk milk samples from the positive herds, test sensitivity (identical to the mean sensitivity of the ELISA and Q-PCR tests considered separately) would be 163/482 (34\%). However, a more practical definition of sensitivity as described in Materials and Methods is the sensitivity of the bulk-tank milk testing for detection of JD taking into consideration that 2 tests were performed on every bulk milk sample. This was calculated by dividing the total number of JD-positive bulk-tank results (whether by ELISA, Q-PCR, or both tests) by the total number of bulk-tank milk samples tested from the JD-positive herds. In total, 138 JD-positive bulk-tank samples were found among the 241 bulk tank samples from the 67 positive herds; 25 were both ELISA and Q-PCR positive, 67 ELISA were positive only, and 46 were PCR-positive only. Therefore, the sensitivity of a single bulk-tank sample test for detection of JD was $138 / 241(57 \%)$.

\section{Estimates of True-Negative JD Status for $100 \%$ Test-Negative Herds}

Because the bulk-tank sample test sensitivity for detection of JD was $57 \%$, the probability of false-negative results on a single negative tank test was $(1-0.57)$ $=0.43$. The probability of true-negative JD status depending on the number of bulk-tank milk samples tested from any given farm was calculated as described earlier. The true-negative probabilities are shown in Table 3. For example, for farms with 1 bulk tank, 2 samples collected 1 mo apart, with both samples testing negative (by both ELISA and Q-PCR) for JD, the true-negative probability was $\left[1-(0.43)^{2}\right]=(1-0.18)$ $=82 \%$. For farms with at least 2 bulk tanks, at least 4 samples tested, with all results negative for JD, the true-negative probability was at least $97 \%$.

Some dairy producers with positive bulk tank results contacted 2 of the authors (D. Wilson and K. Rood) regarding follow-up testing. Individual cow testing for JD in herds with positive bulk tank results always detected JD-positive cows; this included herds with $2 / 48,2 / 14$, and $1 / 10$ positive bulk-tank test results.

Table 2. Agreement between Johne's disease (JD) ELISA and quantitative real-time PCR (Q-PCR) tests concurrently performed on 241 bulk-tank samples from 67 dairy herds with at least 1 positive JD test result

\begin{tabular}{lccc}
\hline & \multicolumn{2}{c}{ JD ELISA result } & \\
\cline { 2 - 3 } $\begin{array}{l}\text { JD Q-PCR } \\
\text { result }\end{array}$ & Positive & Negative & Total \\
\hline Positive & 25 & 46 & 71 \\
Negative & 67 & 103 & 170 \\
Total & 92 & 149 & 241 \\
\hline
\end{tabular}


Table 3. Probability of true-negative Johne's disease (JD) status for 103 dairy herds depending on the number of bulk tank milk samples tested, where all samples tested negative for Mycobacterium avium ssp. paratuberculosis

\begin{tabular}{lcc}
\hline & & $\begin{array}{c}\text { Probability } \\
\text { that herd is } \\
\text { truly JD } \\
\text { No. of tank samples } \\
\text { (all tested negative) }\end{array}$ \\
\hline $1^{1}$ & Farms, & 57 \\
2 & 25 & 82 \\
3 & 63 & 92 \\
4 & 2 & 97 \\
5 or more & 11 & $>99$ \\
\hline
\end{tabular}

${ }^{1}$ Every farm was intended to have all tanks sampled twice each, but some farms had only one sample from their single tank, and a few others had one of their multiple tanks only single-sampled. Therefore, odd numbers were possible.

\section{DISCUSSION}

Approximately $40 \%$ of dairy herds in Utah and parts of surrounding states tested positive for JD. These results are similar to other recent estimates of herd-level JD prevalence in the US, supporting the speculation that the proportion of herds infected is increasing over the last 15 to $20 \mathrm{yr}$ (Tiwari et al., 2006; Tavornpanich et al., 2008). However, the prevalence detected was 3 times that from a recent report that $13 \%$ of dairy herds in the western US were positive (USDA APHIS, 2008). The overriding characteristic of prevalence estimates for JD in North America is that data are lacking; little testing for paratuberculosis is carried out in dairy herds (W. Hess, Utah Department of Agriculture and Food, Salt Lake City, UT, personal communication; USDA APHIS, 2008). This lack of data regarding JD prevalence is also evident in Europe. A recent review of the European literature concluded that "prevalences of MAP would have to be guesstimates based on available data," and "there is a continuing need for well-designed studies of the prevalence of MAP infections" (Nielsen and Toft, 2009). The continuing increase in proportion of herds and cows within herds infected with JD suggests that current control programs, at least as applied, are not effective. This is not surprising considering that testing for the disease is not implemented on so many farms.

Test sensitivity of a single bulk-tank milk sample was $57 \%$, resulting in a high probability that a herd was truly negative if 4 or more bulk-tank samples from each tank on a farm were test-negative (2 tests were performed on each sample, an ELISA and a Q-PCR). Based on the results of the present study, the recommendation for bulk-tank JD testing using these tests is that a dairy herd should not be considered negative for JD until after at least 4 bulk tank samples have all tested negative. Any positive test result suggests strongly that the herd is infected with JD; this is a highly specific test. However, if a dairy producer is reluctant to progress to individual cow testing for JD after 1 positive bulk-tank result, additional bulk tank tests could be done until another positive result is found.

The cut-off points of the 2 JD tests used in this study were designed to maintain high specificity (avoidance of false-positives), while attempting to minimize loss of sensitivity. Bulk-tank milk testing for diseases is always affected by disease prevalence, intermittent shedding of pathogens, and possible increased dilution of pathogens or antibodies if uninfected cows produce more average milk than infected cows.

The overall agreement between the bulk tank ELISA and Q-PCR assay was greater than $75 \%$, but agreement was just over $50 \%$ within JD-positive herds. Imperfect agreement between the 2 assays on positive bulk-tank samples is expected given that the 2 assays detect different targets. The ELISA targets antibodies in the bulk tank, which can only come from cow milk and are the result of a variable and delayed humoral response to MAP infection (Stabel, 2006). On the other hand, the Q-PCR assay targets the presence of MAP DNA, which can come from milk or environmental contamination and is generally shed in manure earlier during the progression of paratuberculosis (Sweeney et al., 2006). Consequently, agreement between the 2 tests in infected herds would be affected by factors other than test performance, including disease prevalence, proportion of infected animals in later stages of disease, and milking hygiene.

The utilization of bulk milk sample testing is a practical way to screen dairy herds for presence of JD. Milk sampling is markedly more convenient than fecal or blood sample testing for any disease of dairy cattle. Studies on the use of individual cow milk tests for accuracy, practicality, and together with management strategies, their effectiveness in reducing the prevalence of JD in dairy herds are needed.

\section{CONCLUSIONS}

Testing of dairy herds for JD has been decreasing and is poorly utilized in the dairy industry. Using a repeated-testing strategy, such as collecting monthly samples, on bulk-tank milk is a practical and convenient way to monitor for the presence of JD in dairy herds. Studies on the use of individual cow milk tests for JD as part of a strategy to reduce the disease in dairy herds are needed. 


\section{ACKNOWLEDGMENTS}

This work was funded by grants from Utah State University (Logan) and Utah State University Supplemental Extension Funds. We appreciate the cooperation of the owners of the participating dairy farms and the milk buyer personnel who recruited the participants and collected the milk samples.

\section{REFERENCES}

Biswas, P., W. Foshaug, H. Donohue, J. Lombard, and T. Byrem. 2008. Bulk milk testing for Johne's disease detection at the farm level. Page 27 in Proc. Johne's Disease Integrated Program (JDIP) 4th Ann. Conf., East Lansing, MI.

Chi, J., J. A. VanLeeuwen, A. Weersink, and G. P. Keefe. 2002. Direct production losses and treatment costs from bovine viral diarrhoea virus, bovine leukosis virus, Mycobacterium avium subspecies paratuberculosis, and Neospora caninum. Prev. Vet. Med. 55:137-153.

Clark, D. L., J. J. Koziczowski, R. P. Radcliff, R. A. Carlson, and J. L. Ellingson. 2008. Detection of Mycobacterium avium subspecies paratuberculosis: Comparing fecal culture versus serum enzymelinked immunosorbent assay and direct fecal polymerase chain reaction. J. Dairy Sci. 91:2620-2627.

Collins, M. T., D. C. Sockett, W. J. Goodger, T. A. Conrad, C. B. Thomas, and D. J. Carr. 1994. Herd prevalence and geographic distribution of, and risk factors for, bovine paratuberculosis in Wisconsin. J. Am. Vet. Med. Assoc. 204:636-641.

Lombard, J. E., B. A. Wagner, R. L. Smith, B. J. McCluskey, B. N. Harris, J. B. Payeur, F. B. Garry, and M. D. Salman. 2006. Evaluation of environmental sampling and culture to determine Mycobacterium avium subspecies paratuberculosis distribution and herd infection status on US dairy operations. J. Dairy Sci. 89:4163-4171.

McKenna, S. L., G. P. Keefe, H. W. Barkema, J. McClure, J. A. Vanleeuwen, P. Hanna, and D. C. Sockett. 2004. Cow-level prevalence of paratuberculosis in culled dairy cows in Atlantic Canada and Maine. J. Dairy Sci. 87:3770-3777.

Merkal, R. S., D. L. Whipple, J. M. Sacks, and G. R. Snyder. 1987. Prevalence of Mycobacterium paratuberculosis in ileocecal lymph nodes of cattle culled in the United States. J. Am. Vet. Med. Assoc. 190:676-680.

Nielsen, S. S., and N. Toft. 2009. A review of prevalences of paratuberculosis in farmed animals in Europe. Prev. Vet. Med. 88:1-14.

Ott, S. L., S. J. Wells, and B. A. Wagner. 1999. Herd-level economic losses associated with Johne's disease on US dairy operations. Prev. Vet. Med. 40:179-192.
Pearce, B. H., G. T. Fosgate, M. P. Ward, A. J. Roussel, B. Norby, S. Tavornpanich, and D. Ellis. 2008. Comparison of three methods of surveillance with application to the detection of Johne's disease seropositivity in Texas cattle. Prev. Vet. Med. 86:1-7.

Sockett, D. C. 1996. Johne's disease eradication and control: Regulatory implications. Vet. Clin. North Am. Food Anim. Pract. $12: 431-440$

Stabel, J. R. 1998. Johne's disease: A hidden threat. J. Dairy Sci. $81: 283-288$.

Stabel, J. R. 2006. Host responses to Mycobacterium avium ssp. paratuberculosis: A complex arsenal. Anim. Health Res. Rev. 7:61-70.

Sweeney, R. W., R. H. Whitlock, S. McAdams, and T. Fyock. 2006. Longitudinal study of ELISA seroreactivity to Mycobacterium avium ssp. paratuberculosis in infected cattle and culture-negative herd mates. J. Vet. Diagn. Invest. 18:2-6.

Tavornpanich, S., W. O. Johnson, R. J. Anderson, and I. A. Gardner. 2008. Herd characteristics and management practices associated with seroprevalence of Mycobacterium avium subsp paratuberculosis infection in dairy herds. Am. J. Vet. Res. 69:904-911.

Tiwari, A., J. A. VanLeeuwen, I. R. Dohoo, G. P. Keefe, and A. Weersink. 2008. Estimate of the direct production losses in Canadian dairy herds with subclinical Mycobacterium avium subspecies paratuberculosis infection. Can. Vet. J. 49:569-576.

Tiwari, A., J. A. VanLeeuwen, I. R. Dohoo, H. Stryhn, G. P. Keefe, and J. P. Haddad. 2005. Effects of seropositivity for bovine leukemia virus, bovine viral diarrhoea virus, Mycobacterium avium subspecies paratuberculosis, and Neospora caninum on culling in dairy cattle in four Canadian provinces. Vet. Microbiol. 109:147-158.

Tiwari, A., J. A. VanLeeuwen, S. L. McKenna, G. P. Keefe, and H. W. Barkema. 2006. Johne's disease in Canada Part I: Clinical symptoms, pathophysiology, diagnosis, and prevalence in dairy herds. Can. Vet. J. 47:874-882.

USDA APHIS. 2008. APHIS Info Sheet. Johne's disease on U.S. dairies, 1991-2007. USDA-APHIS, Fort Collins, CO.

van Weering, H., G. van Schaik, A. van der Meulen, M. Waal, and K. van Maanen. 2007. Diagnostic performance of the Pourquier ELISA for detection of antibodies against Mycobacterium avium subspecies paratuberculosis in individual milk and bulk milk samples of dairy herds. Vet. Microbiol. 125:49-58.

Wilson, D. J., C. Rossiter, H. R. Han, and P. M. Sears. 1993. Association of Mycobacterium paratuberculosis infection with reduced mastitis, but with decreased milk production and increased cull rate in clinically normal dairy cows. Am. J. Vet. Res. 54:1851-1857.

Wilson, D. J., C. M. Rossiter, H. R. Han, and P. M. Sears. 1995. Financial effects of Mycobacterium paratuberculosis on mastitis, milk production and cull rate in clinically normal cows. Agripractice 16:12-18. 